cross-section for gamma ray excitation of lead is distinctly smaller than that of copper. The energy determination of emitted gamma rays is very interesting in this respect.

A detailed report of this work will be published shortly in Proc. Phys. Math. Soc. Jap.

Physical Institute,

Osaka Imperial University, Osaka.

Jan. 1.

${ }^{1}$ Proc. Roy. Soc., A, 150, 637 (1935).

2 Nature, 137, 398 (1936), and Proc. Phys. Math. Soc. Jap., 18, 115 (1936).

${ }^{3}$ Phys. Rev., 45, 586 (1934).

' Nature, 138, 841 (1936).

s Proc. Roy. Soc., A, 155, $43+$ (1936).

\section{Mechanical Tracer for Electron Trajectories}

The paths of electrons in vacuum devices have been in recent years objects of intensive study. The problem involves in general the solution of a partial differential equation for the field, and if the field is known, the solution of an ordinary differential equation for the motion of the electron. The first problem is usually solved experimentally, by constructing a large-scale model of the device, which is immersed in an electrolyte, and exploring the field with a probe. The second step is either to construct the electron paths by some graphical method in a map of the potential lines, or to introduce the data derived from probe measurements into certain approximative differential equations, as developed by H. Busch, W. Glaser, O. Scherzer et al.

An apparatus has been designed which makes it possible to trace the electron paths without mapping the potential field, and without calculation. Its

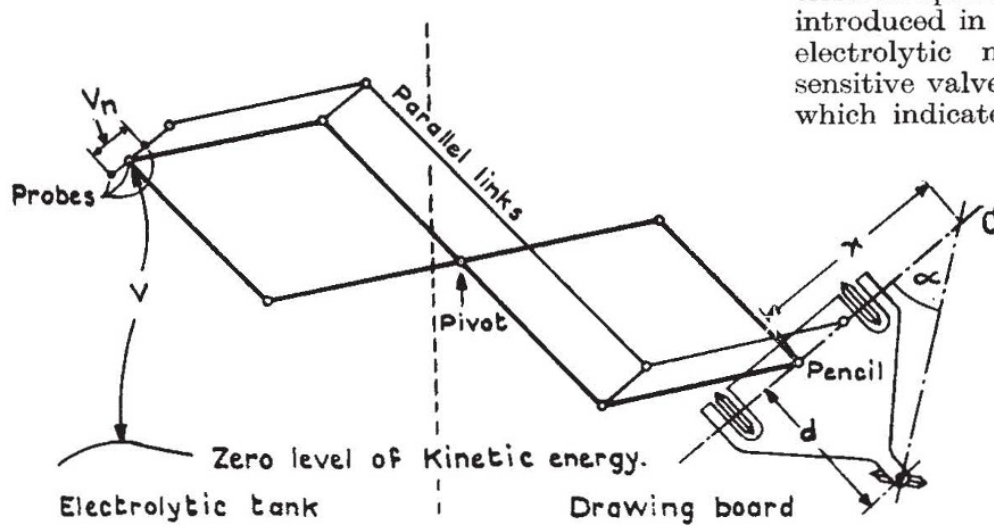

Fig. 1.

principle, in the case of space-charge free electrostatic fields and plane electron paths, is as follows :

The curvature radius of the electron path is $r=2 V / E_{n}$, where $V$ is the potential drop in which the electron has acquired its energy and $E_{n}$ the field intensity normal to the path. In the electrolytic model the electron is represented by a probe and $V$ is its potential with respect to the zero level of electron energy. $E_{n}$ is measured by side probes, placed closely at either side of the main probe, at equal distances. The connecting line of these probes is kept always perpendicular to the path by the mechanism shown in Fig. 1.
The pencil tracing the electron path on a drawing board is carried by a trolley with three sharpedged wheels. The pencil is connected with the main probe by means of a pantograph, and the trolley is linked with the side probes in such a way that their connecting line is always parallel to the leading wheels of the trolley, and therefore perpendicular to the path.

The trolley is steered by its third wheel, which can turn around a vertical axis. If the steering angle is $\alpha$, the curvature radius of the pencil trace is $r=d \cot \alpha$. The pencil draws the true electron path if $\tan \alpha=k \cdot V_{n} / V$, where $V_{n}$ is the voltage between the side probes and $k$ is a constant of the device. This steering angle is determined without any calculation by a special bridge, shown in Fig. 2 :

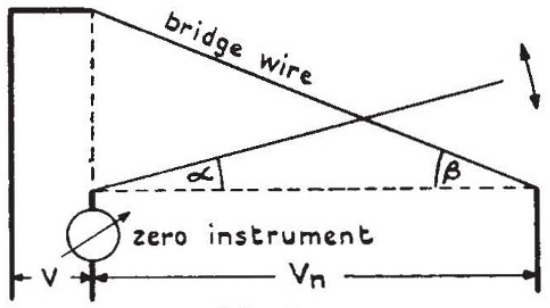

Fig. 2.

This bridge is set to zero if $\tan \alpha=\tan \beta . V_{n} / V$. If $\tan \beta$ is made equal to $k$, the steering angle is found by balancing $V$ on a second bridge and simultaneously balancing the 'tangent bridge'. This angle is transmitted to the steering wheel automatically by means of a differential gear and parallel links. The trolley is moved on in small steps, keeping the bridges in balance all the time, and the pencil traces the path of the electron (or of any other charged particle).

In order to obtain the high accuracy required in electron-optical work, an improvement has been introduced in the bridge method as hitherto used in electrolytic model experiments. An extremely sensitive valve-wattmeter is used as zero instrument, which indicates zero, not if the slider of the bridge which indicates zero, not if the slider of the bridge has the same potential as the probe (a condition which cannot be fulfilled in general), but if the voltage vector between them is perpendicular to a fixed voltage chosen as 'base'. Thus one timecomponent of the potential vectors is sorted out. This has the advantage that it not only reduces the random error to negligible proportions, but also eliminates at the same time the systematic error, introduced by the polarization capacities at the electrodes.

The first apparatus of this particular kind has recently been completed in the Research Laboratories of the British Thomson-Houston Co. Ltd, Rugby. I wish to acknowledge my grateful thanks to my colleagues in the Research Laboratory, and to Mr. $H$. Warren, chief of the Laboratory, and the directors of the B.T.H. Co. Ltd. for permission to publish this note.

Research Laboratory,

D. Gabor.

British Thomson-Houston Co. Ltd., Rugby.

Jan. 16 